\title{
Comportamento reológico de iogurte sabor bacuri: efeitos da temperatura e do teor de gordura
}

\author{
Rheological behavior of bacuri yogurt: effects of temperature \\ and fat content
}

\author{
Adriano Cesar Calandrini Braga ${ }^{1 *}$ (1), Priscila Dias Saldanha Ramos ${ }^{1}$, \\ Raquel Pinto da Costa ${ }^{1}$, Licia Amazonas Calandrini Braga ${ }^{2}$
}

${ }^{1}$ Universidade do Estado do Pará (UEPA) Departamento de Tecnologia de Alimentos (DETA), Castanhal/PA - Brasil ${ }^{2}$ Instituto Federal de Educação, Ciência e Tecnologia do Pará (IFPA), Departamento de Ensino (DEPE),

Castanhal/PA - Brasil

${ }^{*}$ Corresponding Author: Adriano Cesar Calandrini Braga, Universidade do Estado do Pará (UEPA), Departamento de Tecnologia de Alimentos (DETA), Campus XX, Rua Pedro Porpino da Silva, 1181, Bairro São José,

CEP: 68745-000, Castanhal/PA - Brasil. e-mail: accbraga@uepa.br

Cite as: Braga, A. C. C., Ramos, P. D. S., Costa, R. P., \& Braga, L. A. C. (2021). Rheological behavior of bacuri yogurt: effects of temperature and fat content. Brazilian Journal of Food Technology, 24, e2020170.

https://doi.org/10.1590/1981-6723.17020

\begin{abstract}
Resumo
O objetivo deste estudo foi a elaboração e a caracterização reológica de um iogurte sabor bacuri com reduzido teor de gordura. Foram elaborados três iogurtes a partir dos tipos de leite UHT integral, semidesnatado e desnatado. As análises reológicas foram realizadas em quatro diferentes temperaturas $\left(5^{\circ} \mathrm{C}, 15^{\circ} \mathrm{C}, 30^{\circ} \mathrm{C}\right.$ e $\left.45^{\circ} \mathrm{C}\right)$ e os dados experimentais foram ajustados ao modelo de Ostwald-de-Waelle. A relação entre temperatura e viscosidade aparente foi descrita por uma equação tipo Arrhenius. Os valores de tensão de cisalhamento e viscosidade aparente dos iogurtes diminuíram com o aumento da temperatura, bem como com a redução do teor de gordura. O modelo utilizado mostrou-se adequado para descrever o comportamento reológico dos iogurtes, segundo os parâmetros estatísticos avaliados. Os baixos valores obtidos para o índice de comportamento indicaram um comportamento característico de fluidos pseudoplásticos $(\mathrm{n}<1)$. A equação tipo Arrhenius descreveu de modo satisfatório o efeito da temperatura sobre a viscosidade aparente. Os valores da energia de ativação ( $E_{\text {att }}$ ) foram de $2,66 \mathrm{kcal} \mathrm{g} \mathrm{mol}^{-1}$, $3,81 \mathrm{kcal} \mathrm{g} \mathrm{mol}^{-1}$ e $3,93 \mathrm{kcal} \mathrm{g} \mathrm{mol}^{-1}$, respectivamente, para as amostras de iogurte integral, semidesnatado e desnatado. Na caracterização físico-química, os teores de gordura se mostraram próximos para os iogurtes desnatado e semidesnatado, não havendo diferença significativa. O iogurte semidesnatado e o desnatado podem ser considerados com reduzido teor de gordura, pois sofreram uma diminuição no teor de lipídios de $75 \%$ e $79,1 \%$, respectivamente.
\end{abstract}

Palavras-chave: Platonia insignis Mart.; Temperatura; Teor de gordura; Viscosidade aparente; Fermentação.

\section{Abstract}

This work aimed to evaluate the preparation and rheological characterization of a bacuri yogurt with reduced-fat content. Three types of yogurts from UHT whole milk, semi-skimmed and skimmed were elaborated. The rheological 
analyses were performed in four different temperatures $\left(5^{\circ} \mathrm{C}, 15^{\circ} \mathrm{C}, 30^{\circ} \mathrm{C}\right.$ and $\left.45^{\circ} \mathrm{C}\right)$ and the experimental data were adjusted for Power Law model. The relation of temperature with apparent viscosity was described by Arrhenius equation. The values of yield stress and apparent viscosity for yogurts decreased with increasing temperature as well as with fat content. The model used was adequate to describe the rheological behavior of the yogurts according to the statistics parameters evaluated. The low values obtained for the behavior index indicated a characteristic behavior of pseudoplastic fluids $(n<1)$. The Arrhenius equation described properly the effect of temperature in apparent viscosity. The values of activation energy were $2.66 \mathrm{kcal} \mathrm{g} \mathrm{mol}^{-1}, 3.81 \mathrm{kcal} \mathrm{g} \mathrm{mol}^{-1}$ and $3.93 \mathrm{kcal} \mathrm{g} \mathrm{mol}^{-1}$, with respect to the samples of whole yogurt, semi-skimmed and skimmed, respectively. Regarding the physicochemical characterization, the fat levels were similar for the skimmed and semi-skimmed yogurts, demonstrating no significant difference. The semi-skimmed and skimmed yogurts were considered light or with reduced-fat content, thus determining a decrease in values of $75 \%$ and $79.1 \%$, respectively.

Keywords: Platonia insignis Mart.; Temperature; Fat content; Apparent viscosity; Fermentation.

\section{Introdução}

Em todo o mundo, o número de produtos lácteos ofertados ao consumidor tem aumentado consideravelmente e, dentro da classe dos leites fermentados, o iogurte se destaca pela sua grande disponibilidade e aceitação no mercado (Özer \& Kirmaci, 2010; Soares et al., 2011). O consumo de iogurtes é estimulado pela busca do consumidor por produtos que promovam benefícios à saúde, uma vez que é um alimento rico em proteínas, ácido fólico, vitamina A, vitaminas do complexo B e sais minerais, e que contém fermentos probióticos, que podem, em muitos casos, ser considerados "funcionais", auxiliando no controle e na modulação de várias funções orgânicas, e contribuindo para a manutenção da saúde e a redução do risco de aparecimento de determinadas doenças crônicas (Borges, 2001; Chandan et al., 2006).

A qualidade do produto é de grande importância na sua aceitação e esta é influenciada, principalmente, por sua consistência e/ou viscosidade (Mathias et al., 2013). Outra característica igualmente importante na definição da qualidade do iogurte é a separação da fase solúvel, denominada de sinérese (separação do soro) (Lee \& Lucey, 2010). Dois fatores são de grande importância nas características de textura do produto: a adição de sólidos e o tratamento térmico (Antunes et al., 2004). O controle da viscosidade do produto em nível industrial é feito por meio do aumento do teor de sólidos presentes pela adição de leite em pó ou soro de leite em pó, podendo, ainda, serem adicionados espessantes com esta finalidade, atribuindo uma textura mais firme (Mathias et al., 2013).

A manutenção da estabilidade de um produto ou sua estrutura durante a estocagem é afetada por suas propriedades reológicas, principalmente para emulsões e outras dispersões. Na ciência dos alimentos, a reologia é usada para o estudo da estrutura dos alimentos como resposta à aplicação de uma força ou deformação (Steffe, 1996).

Em determinadas condições (moderada taxa de deformação, conteúdo de gordura inferior a $40 \%$ e temperaturas superiores a $40^{\circ} \mathrm{C}$, nas quais a gordura está líquida e não ocorre aglutinação pelo frio), leite, leite desnatado e creme são fluidos com propriedades newtonianas. Todavia, leite cru e cremes exibem propriedades reológicas de fluidos não newtonianos, quando são submetidos a condições em que é possível a solidificação dos glóbulos de gordura (abaixo de $40{ }^{\circ} \mathrm{C}$ e baixas taxas de deformação, 0,1 a $20 \mathrm{~s}^{-1}$ ) (Fox et al., 2015).

A demanda por produtos light (conteúdo reduzido de determinado componente) tem crescido muito e tende a aumentar ainda mais. Na primeira década dos anos 2000, o mercado desse tipo de produto registrou aumento de $870 \%$, segundo a Associação Brasileira da Indústria de Alimentos Dietéticos e para Fins Especiais ABIAD (Ribeiro et al., 2010). No segmento de iogurtes, há uma procura crescente por itens com baixo teor de gordura que apresentem textura diferente em comparação a produtos integrais (Teles \& Flôres, 2007b). 
Para uma melhor aceitação do produto do ponto de vista sensorial, são adicionados preparos à base de frutas, a fim de somar suas características, especialmente de aroma e sabor, ao iogurte.

Nesse contexto, o Brasil se destaca por sua considerável biodiversidade de frutíferas, sendo alta a potencialidade para o aproveitamento de seus frutos (Fernandes et al., 2010). O bacuri (Platonia insignis Mart.) é uma das frutas mais populares na Região Norte e nos estados vizinhos à região amazônica. Possui altos teores de fibras totais, que exercem efeitos positivos sobre a fisiologia intestinal e do cólon, favorecendo a saciedade e a digestão. Apresenta minerais, como zinco, cálcio (garante a saúde dos ossos e dentes), fósforo e potássio, que são componentes ou ativadores/reguladores de muitas enzimas, além de estarem envolvidos no processo de crescimento e desenvolvimento corporal. Possui vitaminas, como a vitamina $\mathrm{B}_{2}$, que auxilia na produção de energia para o organismo (Rogez et al., 2004; Silva et al., 2010). O estado do Pará apresenta destaque no cenário nacional como produtor de bacuri, fato que, aliado às excelentes características sensoriais e nutricionais da fruta, favorece sua utilização na elaboração de diversos derivados (Souza et al., 2001).

O presente trabalho teve por objetivo a elaboração e a caracterização reológica de um iogurte sabor bacuri com reduzido teor de gordura, como forma de diversificar a cadeia produtiva do fruto e agregar maior valor às matérias-primas características da Amazônia, aumentando a disponibilidade de dados acerca de produtos à base de frutas da região, em geral, pouco difundidos em nível nacional e, sobre os quais, há escassos estudos na literatura. A obtenção de dados é importante, pois pode ensejar e/ou servir como base de projetos de tubulações e de bombas, e sistemas de agitação e de mistura, que se adequem às necessidades específicas do produto. Para isso, foi avaliada a influência do teor de gordura e da temperatura sobre o comportamento reológico do iogurte, além da sua caracterização físico-química.

\section{Material e métodos}

\subsection{Processo de elaboração dos iogurtes}

Foram empregados, no preparo dos produtos, os tipos de leite UHT integral, semidesnatado e desnatado, polpa de bacuri, sacarose (açúcar refinado) e culturas starter de Streptococcus salivarius subsp. thermophilus e Lactobacillus delbrueckii subsp. bulgaricus, adquiridos em estabelecimentos comerciais da cidade de Belém-PA.

Os leites foram aquecidos à temperatura de $45{ }^{\circ} \mathrm{C}$, em quantidades suficientes para preparar cinco litros de cada iogurte. Adicionou-se $3 \% \mathrm{~m} / \mathrm{m}$ da cultura láctica ativa a cada leite, bem como $15 \% \mathrm{~m} / \mathrm{m}$ de sacarose, de acordo com os resultados obtidos em testes preliminares. Posteriormente, os leites acrescidos do inóculo foram incubados em estufa a $43 \pm 2{ }^{\circ} \mathrm{C}$ para que ocorresse o processo de fermentação.

Atingido o valor desejado de acidez $(0,87 \%$ a $0,90 \%$ de ácido láctico), foi feito um resfriamento inicial a uma temperatura de aproximadamente $20^{\circ} \mathrm{C}$ e promoveu-se a quebra do coágulo por agitação manual durante 30 segundos, de forma padronizada. Em seguida, os produtos foram resfriados à temperatura de $10{ }^{\circ} \mathrm{C}$ para a redução da atividade metabólica da cultura.

A polpa de bacuri foi adicionada numa proporção de $15 \%$ para os três produtos. A homogeneização dos iogurtes foi realizada manualmente e foram então envasados em embalagens de polietileno de $200 \mathrm{~mL}$, sendo armazenados em refrigerador à temperatura de $6{ }^{\circ} \mathrm{C}$ a $8{ }^{\circ} \mathrm{C}$, aproximadamente. Depois de elaborados, os iogurtes foram codificados da seguinte forma: iogurte de bacuri integral - I.I, iogurte de bacuri semidesnatado - I.S.D e iogurte de bacuri desnatado - I.D.

\subsection{Caracterização físico-química dos iogurtes}

Foram realizadas análises de umidade, acidez (\% ácido láctico), cinzas, proteínas e gordura, de acordo com a metodologia da AOAC - Association of Official Analytical Chemists (2016). Todas as análises foram realizadas em triplicata e os resultados foram submetidos à análise de variância (ANOVA), e as médias 
comparadas entre si pelo teste de Tukey ao nível de $5 \%$ de significância $(p<0,05)$, por meio do programa Statistica 7.0 (Statsoft - USA).

\subsection{Análise reológica dos iogurtes}

Os ensaios reológicos foram conduzidos em delineamento inteiramente casualizado, com duas repetições, mediante o emprego de um viscosímetro de cilindros concêntricos Brookfield, modelo LVDV II, acoplado a um banho termostático Haake B3, utilizando o cilindro interno de especificação DIN S87 e o programa de ajuste Wingather ${ }^{\circledR}$. Para cada um dos ensaios, foram utilizados $8 \mathrm{~mL}$ de amostra, sendo analisada em quatro diferentes temperaturas: $5^{\circ} \mathrm{C}, 15^{\circ} \mathrm{C}, 30^{\circ} \mathrm{Ce} 45^{\circ} \mathrm{C}$. As velocidades rotacionais do cilindro interno estiveram na faixa de 5 a $100 \mathrm{rpm}$ e a taxa de deformação ficou entre $6,4 \mathrm{e} 129 \mathrm{~s}^{-1}$.

Os dados de tensão de cisalhamento e taxa de deformação obtidos experimentalmente foram ajustados ao modelo reológico de Ostwald-de-Waelle por meio do software Statistica 7.0 (Statsoft - USA), sendo o ajuste avaliado pelos parâmetros estatísticos coeficiente de correlação $\left(\mathrm{R}^{2}\right)$ e qui-quadrado $\left(\chi^{2}\right)$, obtido da Equação 1, e o fator bias $\left(\mathrm{B}_{\mathrm{f}}\right)$, pela Equação 2.

$\chi^{2}=\sum \frac{\left(\mathrm{V}_{\mathrm{Exp}}-\mathrm{V}_{\mathrm{Pred}}\right)^{2}}{\mathrm{~N}-\mathrm{n}}$

$\mathrm{B}_{\mathrm{f}}=10^{\left[\sum\left(\mathrm{V}_{\mathrm{Exp}}-\mathrm{V}_{\text {Pred }}\right)^{2} / \mathrm{N}\right]}$

Em que: $\mathrm{V}_{\text {Exp }}=$ Valores obtidos experimentalmente; $\mathrm{V}_{\text {Pred }}=$ Valores preditos pelo ajuste do modelo; $\mathrm{N}$ = número de observações; $\mathrm{n}$ = número de parâmetros do modelo.

A viscosidade aparente (Equação 3) dos iogurtes de bacuri foi calculada de acordo com os parâmetros reológicos do modelo de Ostwald-de-Waelle (Equação 4): índice de consistência (K) e índice de comportamento ao escoamento (n). Substituindo a equação do modelo na Equação 3, obtém-se a Equação 5, que foi utilizada para o cálculo de $\eta_{\mathrm{Ap}}$ das amostras analisadas.

$\eta_{\mathrm{Ap}}=\frac{\tau}{\dot{\gamma}}$
$\tau=\mathrm{K} \times \dot{\gamma}^{\mathrm{n}}$
$\eta_{\mathrm{Ap}}=\frac{\mathrm{K} \times \dot{\gamma}^{\mathrm{n}}}{\dot{\gamma}}$

Em que: $\eta_{\mathrm{Ap}}=$ viscosidade aparente (Pa s); $\tau=$ tensão de cisalhamento; $(\mathrm{Pa}) ; \gamma=$ taxa de deformação $\left(\mathrm{s}^{-1}\right)$;

$\mathrm{K}=$ índice de consistência $\left(\mathrm{Pa} \mathrm{s}^{\mathrm{n}}\right) ; \mathrm{n}=$ índice de comportamento ao escoamento (adimensional).

O efeito da temperatura sobre a viscosidade aparente foi avaliado mediante uma equação tipo Arrhenius (Equação 6) (Silva et al., 2005).

$\eta_{\mathrm{Ap}}=\eta_{0} \times \exp \left(\frac{E_{\mathrm{at}}}{\mathrm{RT}}\right)$

Em que: $\eta_{\mathrm{Ap}}=$ viscosidade aparente $(\mathrm{Pa} \mathrm{s}) ; \eta_{0}=$ Constante $(\mathrm{Pa} \mathrm{s}) ; \mathrm{E}_{\mathrm{at}}=$ Energia de ativação para escoamento viscoso $\left(\mathrm{kcal} \mathrm{g} \mathrm{mol}^{-1}\right) ; \mathrm{R}=$ Constante dos gases $\left(1,987 \times 10^{-3} \mathrm{kcal} \mathrm{g} \mathrm{mol}^{-1} \mathrm{~K}^{-1}\right) ; \mathrm{T}=$ Temperatura absoluta (K).

\subsection{Análise estatística}

Os resultados foram submetidos à Análise de Variância (ANOVA), ao nível de 5\% de significância $(p<0,05)$, a fim de detectar diferença significativa entre os dados de comportamento reológico nas diferentes temperaturas e teores de gordura utilizados. Quando detectada diferença significativa entre os tratamentos, a 
análise foi complementada com a aplicação do teste de Tukey $(p<0,05)$, por meio do programa Statistica 7.0 (Statsoft - USA).

\section{Resultados e discussão}

\subsection{Caracterização físico-química dos iogurtes}

Os resultados obtidos na caracterização dos iogurtes de bacuri são observados na Tabela 1.

Tabela 1. Composição físico-química dos iogurtes de bacuri integral - I.I, semidesnatado - I.S.D e desnatado - I.D.

\begin{tabular}{cccc}
\hline Análises & I.I & I.S.D & I.D \\
\hline Umidade & $74,83^{\mathrm{b}} \pm 0,015$ & $76,57^{\mathrm{a}} \pm 0,00075$ & $77,46^{\mathrm{a}} \pm 0,029$ \\
\hline Cinzas & $0,72^{\mathrm{a}} \pm 0,033$ & $0,76^{\mathrm{a}} \pm 0,00025$ & $0,75^{\mathrm{a}} \pm 0,019$ \\
\hline Lipídeos & $2,4^{\mathrm{a}} \pm 0,04$ & $0,6^{\mathrm{b}} \pm 0,08$ & $0,5^{\mathrm{b}} \pm 0,03$ \\
\hline Proteínas & $2,53^{\mathrm{a}} \pm 0,013$ & $2,61^{\mathrm{a}} \pm 0,00025$ & $2,58^{\mathrm{a}} \pm 0,05$ \\
\hline Acidez (\% ácido láctico) & $1,04^{\mathrm{a}} \pm 0,08$ & $1,05^{\mathrm{a}} \pm 0,010$ & $0,97^{\mathrm{a}} \pm 0,07$ \\
\hline
\end{tabular}

Médias seguidas por letras iguais nas linhas não diferem entre si pelo teste de Tukey $(p>0,05)$.

A composição do iogurte é similar à do leite, embora se reconheça que há algumas diferenças devido às mudanças ocorridas pelo processo fermentativo das bactérias lácticas sobre a lactose (Ribeiro et al., 2007). Segundo Brasil (2007), os valores de lipídeos variam de 3\% a 5,9\% para o iogurte integral e de $0,6 \%$ a $2,9 \%$ para o semidesnatado, enquanto, para o desnatado, é de no máximo $0,5 \%$. Nos iogurtes de bacuri elaborados, verifica-se que o semidesnatado e o desnatado apresentaram valores de lipídeos dentro da faixa estabelecida pela legislação citada, não demonstrando diferença estatisticamente significativa entre si. Já o iogurte de bacuri integral apresentou um valor de lipídeos abaixo do estabelecido na legislação, podendo esse fato estar relacionado à composição da matéria-prima utilizada.

O iogurte de bacuri semidesnatado apresentou uma redução de $75 \%$ no conteúdo de gordura em comparação ao produto integral, enquanto o iogurte de bacuri desnatado obteve $79,16 \%$ de redução no conteúdo de gordura, em comparação à bebida integral. Desse modo, ambos podem ser classificados como iogurte de bacuri light ou com conteúdo reduzido de gorduras, uma vez que apesentaram redução superior a 25\% nesse componente, em relação ao alimento de referência (Brasil, 2012).

Os valores encontrados para acidez mostram que os iogurtes apresentam valores aproximados, não sendo detectada diferença significativa $(p>0,05)$, e que os três iogurtes encontram-se dentro dos padrões estabelecidos pela legislação vigente (Brasil, 2007), que estabelece a acidez dos leites fermentados entre 0,6 e 2,0 g de ácido láctico/100 g. Da mesma forma, não houve diferença significativa entre os valores de cinzas dos iogurtes.

Com relação às proteínas, observou-se que não houve diferença estatisticamente significativa entre os três tipos de iogurtes elaborados, estando os valores abaixo dos padrões exigidos pela legislação (Brasil, 2007), que é de no mínimo 2,9 g/100 g. Segundo Souza et al. (2001), este fato pode ser atribuído à baixa quantidade de proteínas presentes na polpa de bacuri quando comparada a outras frutas. Logo, a quantidade presente foi insuficiente para equilibrar a perda do teor proteico proveniente do processo fermentativo durante a elaboração do produto. De acordo com Lima et al. (2006), a correção do conteúdo de proteínas pode ser feita por meio da adição de sólidos de origem láctea ao produto, como na forma de leite em pó ou concentrado proteico de soro, a fim de que o teor de proteínas atenda ao estabelecido pela legislação. 


\subsection{Análise reológica}

Os resultados do ajuste dos dados experimentais ao modelo proposto indicaram elevados valores de $\mathrm{R}^{2}$ $(>0,96)$ para todas as amostras analisadas, além de baixos valores de $\chi^{2}$ e um fator bias (Bf) igual a um, sendo, por isso, aceitável para descrever o comportamento reológico dos iogurtes de bacuri. Bezerra (2010) utilizou o modelo de Ostwald-de-Waelle para avaliar os dados reológicos de iogurtes obtidos pela mistura dos leites bubalino e caprino, resultando em valores do coeficiente de determinação $\left(R^{2}\right)$ superiores a 0,95 . Já Dönmez et al. (2017) avaliaram dados reológicos obtidos na avaliação da sinérese de iogurte contendo chá verde e pó de café verde, obtendo valores de coeficiente de determinação maiores que 0,994 , sendo equivalentes aos observados para os iogurtes de bacuri avaliados neste trabalho. A Tabela 2 mostra os parâmetros de ajuste do modelo de Ostwald-de-Waelle para as amostras de iogurte nas quatro temperaturas analisadas.

Tabela 2. Parâmetros do modelo de Ostwald-de-Waelle para as amostras de iogurte de bacuri integral, semidesnatado e desnatado.

\begin{tabular}{|c|c|c|c|c|}
\hline \multicolumn{5}{|c|}{ Iogurte de bacuri integral } \\
\hline Parâmetros & $5^{\circ} \mathrm{C}$ & $15^{\circ} \mathrm{C}$ & $30{ }^{\circ} \mathrm{C}$ & $45^{\circ} \mathrm{C}$ \\
\hline $\mathrm{K}$ & $2,5536^{\mathrm{A}^{*}, \mathrm{~b}^{* *}}$ & $1,4059^{\mathrm{B} *, \mathrm{a}^{* *}}$ & $1,3863^{\mathrm{B}^{*}, \mathrm{a}^{* *}}$ & $1,2416^{\mathrm{B}^{*}, \mathrm{a}^{* *}}$ \\
\hline $\mathrm{n}$ & $0,3265^{\mathrm{A}^{*}, \mathrm{a}^{* *}}$ & $0,3962^{\mathrm{A}^{*}, \mathrm{a}^{* *}}$ & $0,3315^{\mathrm{A}^{*}, \mathrm{a}^{* *}}$ & $0,3310^{\mathrm{A}^{*}, \mathrm{a}^{* *}}$ \\
\hline $\mathrm{R}^{2}$ & 0,9932 & 0,9967 & 0,9940 & 0,9929 \\
\hline$\chi^{2}$ & 0,0530 & 0,0186 & 0,0148 & 0,0137 \\
\hline $\mathrm{B}_{\mathrm{f}}$ & 1,0996 & 1,0339 & 1,0268 & 1,0249 \\
\hline \multicolumn{5}{|c|}{ Iogurte de bacuri semidesnatado } \\
\hline Parâmetros & $5^{\circ} \mathrm{C}$ & $15^{\circ} \mathrm{C}$ & $30^{\circ} \mathrm{C}$ & $45^{\circ} \mathrm{C}$ \\
\hline $\mathrm{K}$ & $3,0862^{\mathrm{A}^{*}, \mathrm{a}^{* *}}$ & $1,1919^{\mathrm{B}^{*}, \mathrm{a}^{* *}}$ & $0,8008^{\mathrm{BC}, \mathrm{b}^{* *}}$ & $0,6399^{C^{*}, b^{* *}}$ \\
\hline $\mathrm{n}$ & $0,2126^{\mathrm{A}^{*}, \mathrm{a}^{* *}}$ & $0,3558^{\mathrm{A}^{*}, \mathrm{a}^{* *}}$ & $0,3519^{\mathrm{A}^{*}, \mathrm{a}^{* *}}$ & $0,3614^{\mathrm{A}^{*}, \mathrm{a}^{* *}}$ \\
\hline $\mathrm{R}^{2}$ & 0,9639 & 0,9902 & 0,9946 & 0,9937 \\
\hline$\chi^{2}$ & 0,0810 & 0,0246 & 0,0056 & 0,0047 \\
\hline $\mathrm{B}_{\mathrm{f}}$ & 1,1560 & 1,0450 & 1,0101 & 1,0085 \\
\hline \multicolumn{5}{|c|}{ Iogurte de bacuri desnatado } \\
\hline Parâmetros & $5^{\circ} \mathrm{C}$ & $15^{\circ} \mathrm{C}$ & $30^{\circ} \mathrm{C}$ & $45^{\circ} \mathrm{C}$ \\
\hline $\mathrm{K}$ & $1,9453^{\mathrm{A}^{*}, \mathrm{c}^{* *}}$ & $1,4483^{\mathrm{B}^{*}, \mathrm{a}^{* *}}$ & $0,7199^{C^{*}, b^{* *}}$ & $0,5743^{\mathrm{C}^{*}, \mathrm{~b}^{* *}}$ \\
\hline $\mathrm{n}$ & $0,2603^{\mathrm{A}^{*}, \mathrm{a}^{* *}}$ & $0,2625^{\mathrm{A}^{*}, \mathrm{~b}^{* *}}$ & $0,3236^{\mathrm{A}^{*}, \mathrm{a}^{* *}}$ & $0,3185^{\mathrm{A}^{*}, \mathrm{a}^{* *}}$ \\
\hline $\mathrm{R}^{2}$ & 0,9773 & 0,9734 & 0,9796 & 0,9815 \\
\hline$\chi^{2}$ & 0,0417 & 0,0283 & 0,0121 & 0,0066 \\
\hline $\mathrm{B}_{\mathrm{f}}$ & 1,0776 & 1,0520 & 1,0218 & 1,0119 \\
\hline
\end{tabular}

*Médias seguidas por letras maiúsculas iguais nas linhas não diferem entre si pelo teste de Tukey $(p>0,05)$.*Médias seguidas por letras minúsculas iguais nas colunas não diferem entre si pelo teste de Tukey $(p>0,05)$.

O índice de consistência (K) é interpretado como espessamento relativo, sendo que, quanto maior o K, maior é a tendência à aceitabilidade do iogurte (Penna et al., 2001). Para as amostras de iogurte de bacuri analisadas, verifica-se que houve diferença estatisticamente significativa $(p<0,05)$ nos valores de $\mathrm{K}$ entre as diferentes temperaturas, ocorrendo uma redução dos valores desse parâmetro com o aumento da temperatura de análise, nas três amostras analisadas. Um comportamento diferente foi encontrado por NajgebauerLejko et al. (2020), na avaliação de mudanças na viscosidade do gel de iogurte após a suplementação com chás verde e de $\mathrm{Pu}$-erh, em que os autores verificaram um comportamento atípico da variação de temperatura sobre os valores do índice de consistência, sendo que $\mathrm{K}$, à temperatura de $8{ }^{\circ} \mathrm{C}$, assumiu o valor de 2,25 , enquanto que a $10{ }^{\circ} \mathrm{C}, 15{ }^{\circ} \mathrm{C}$ e $20^{\circ} \mathrm{C}$, foram obtidos valores superiores a 3,00 , indicando que houve um aumento dos valores de $\mathrm{K}$ com o aumento da temperatura.

Ao avaliar os resultados entre os tratamentos, verifica-se um comportamento diferente, ou seja, para uma mesma temperatura, houve diferença estatisticamente significativa nos valores de $\mathrm{K}$, entre os diferentes 
iogurtes, somente nas temperaturas de $5{ }^{\circ} \mathrm{C}, 30{ }^{\circ} \mathrm{C}$ e $45{ }^{\circ} \mathrm{C}$. Ao analisar o comportamento de $\mathrm{K}$ nas duas maiores temperaturas de análise, é possível perceber que há uma redução dos valores do índice conforme ocorre a redução do teor de gordura do iogurte. Segundo Penna et al. (2006), esse comportamento pode estar relacionado à diminuição do teor de sólidos totais dos iogurtes em função da redução do teor de gordura, o que pode afetar a percepção da textura do produto por parte do consumidor. De modo similar, Ramírez-Sucre \& Vélez-Ruiz (2013) concluíram, em seu estudo sobre a caracterização reológica de iogurte sabor caramelo, que os sistemas com níveis maiores de geleia de caramelo apresentaram valores de $\mathrm{K}$ significativamente maiores que as demais amostras, pelo aumento do teor de sólidos presentes. Bezerra (2010) observou na análise reológica de iogurte obtido pela mistura dos leites bubalino e caprino que o índice de consistência $(\mathrm{K})$ aumentou significativamente, à medida que foi acrescentado leite de búfala, que apresenta maior teor de gordura em comparação ao leite bovino.

Com relação aos valores dos índices de comportamento (n) dos iogurtes elaborados, não houve diferença significativa $(p>0,05)$ entre os resultados das quatro temperaturas avaliadas, bem como entre os diferentes teores de gordura trabalhados. Todas as medidas foram menores que a unidade, em concordância com dados da literatura, corroborando para a classificação dos fluidos analisados como não newtonianos do tipo pseudoplástico $(\mathrm{n}<1)$. Quanto menor o valor do índice de comportamento reológico (n) em relação a um, maior será a pseudoplasticidade da amostra; caso contrário, quanto maior o valor de $\mathrm{n}$ em relação a um, mais características dilatantes o fluido apresenta (Gonçalvez et al., 2005; Teles \& Flôres, 2007a). Segundo Gurjão et al. (2017), do ponto de vista industrial, esse comportamento reflete um aumento na velocidade de fluxo quando o fluido é submetido a estreitamento de tubulação, em operações como o envase, além da possibilidade de intensa homogeneização em processos de mistura. Diversos outros trabalhos com iogurtes apontam para o comportamento pseudoplástico pelos valores obtidos na avaliação de n (Ramírez-Sucre \& Vélez-Ruiz, 2013; Mathias et al., 2013; Dönmez et al., 2017; Sah et al., 2016; NajgebauerLejko et al., 2020; Agyemang et al., 2020), em concordância com o observado na avaliação dos iogurtes de bacuri.

O comportamento reológico dos iogurtes de bacuri integral, semidesnatado e desnatado é visto nos reogramas da Figura 1, nas quatro temperaturas de análise, nos quais os pontos indicam dados experimentais e as linhas, o comportamento esperado, predito pelo modelo de Ostwald-de-Waelle. É possível constatar que, para uma taxa de deformação constante, há diminuição dos valores de tensão de cisalhamento com o aumento da temperatura. Um comportamento semelhante foi observado por Gurjão et al. (2017) na análise do comportamento reológico de iogurte de cajá avaliado nas temperaturas de $20{ }^{\circ} \mathrm{C}, 30{ }^{\circ} \mathrm{C}$ e $40{ }^{\circ} \mathrm{C}$. Segundo Alpaslan \& Hayta (2002), esse efeito pode ser explicado pelo colapso estrutural das amostras devido à força hidrodinâmica gerada e ao aumento do alinhamento das moléculas constituintes.

Ao avaliar os dados de viscosidade aparente obtidos na avaliação dos iogurtes, é possível notar que os valores de $\eta_{\mathrm{Ap}}$ calculados a uma taxa de deformação fixa de $103 \mathrm{~s}^{-1}$ diminuíram com o aumento da temperatura, indicando que houve diferença estatisticamente significativa $(p<0,05)$ nos resultados, como pode ser observado na Tabela 3 , sendo esse comportamento visto em toda a faixa da taxa de deformação trabalhada $\left(6,4\right.$ e $\left.129 \mathrm{~s}^{-1}\right)$. De acordo com Constenla et al. (1989), a redução na viscosidade aparente pode ser atribuída a um aumento nas distâncias intermoleculares, que ocorre em função da expansão térmica causada pelo aumento na temperatura. Najgebauer-Lejko et al. (2020) avaliaram as mudanças na viscosidade de iogurtes acrescidos de chá verde e chá de Pu-erh, e observaram que a viscosidade aparente aumentou à medida que a temperatura subiu de $8{ }^{\circ} \mathrm{C}$ para $10{ }^{\circ} \mathrm{C}$, o que pode estar relacionado à ocorrência de pós-acidificação, na qual as bactérias lácticas continuam a produzir ácido láctico ao longo da vida útil, fato não verificado na avaliação dos iogurtes de bacuri. 

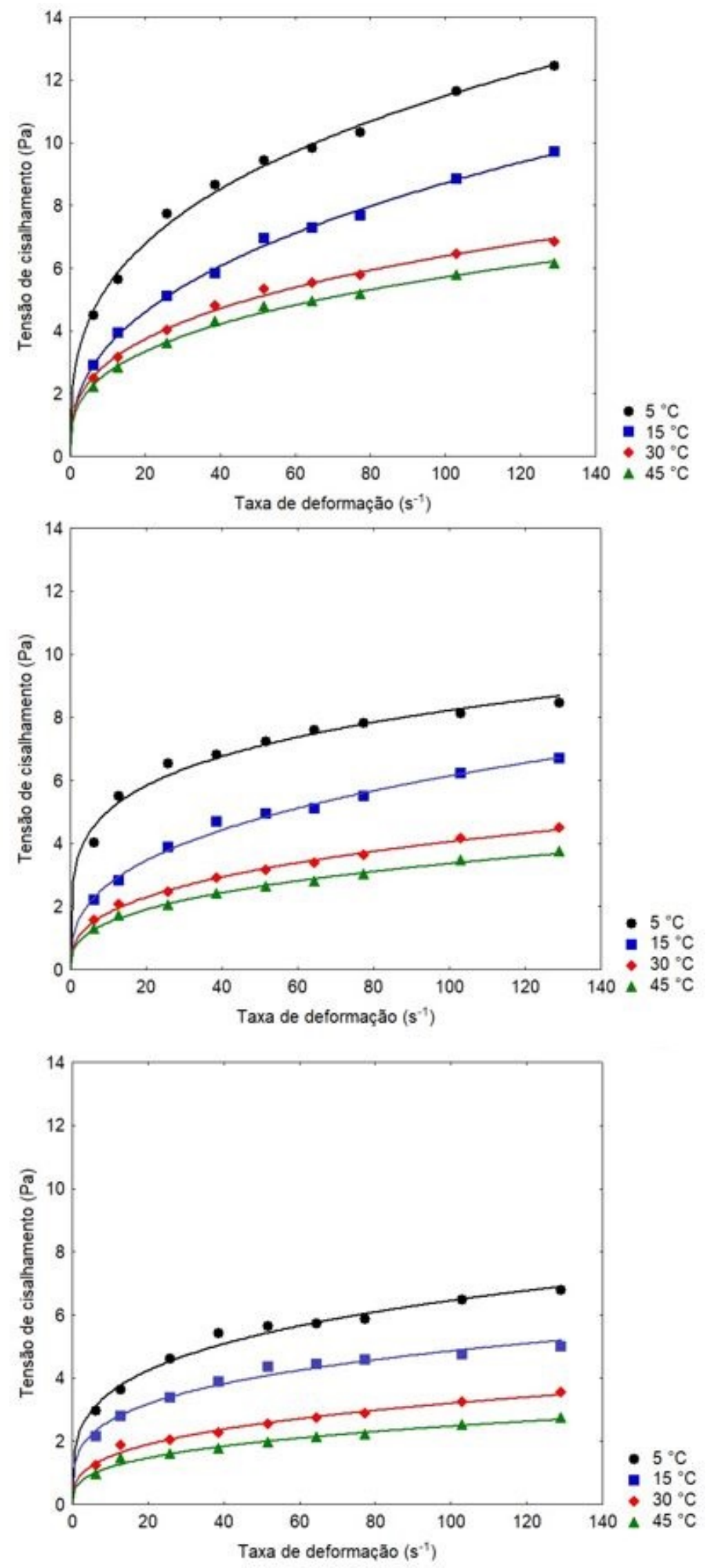

Figura 1. Reogramas dos iogurtes de bacuri integral - I.I (A), semidesnatado - I.S.D (B) e desnatado - I.D (C), pelo modelo de Ostwald-de-Waelle. 
Tabela 3. Viscosidade aparente $\left(\eta_{\mathrm{Ap}}\right)$ das amostras de iogurte de bacuri integral - I.I, semidesnatado - I.S.D e desnatado - I.D nas quatro temperaturas usadas, calculadas à taxa de deformação de $103 \mathrm{~s}^{-1}$.

\begin{tabular}{|c|c|c|c|c|}
\hline \multirow{2}{*}{ Amostras } & \multicolumn{4}{|c|}{$\eta_{A p}(\mathrm{mPa} s)$} \\
\hline & $5^{\circ} \mathrm{C}$ & $15^{\circ} \mathrm{C}$ & $30{ }^{\circ} \mathrm{C}$ & $45^{\circ} \mathrm{C}$ \\
\hline I.I & $112,59^{\mathrm{A}^{*}, \mathrm{a}^{* *}}$ & $85,63^{\mathrm{B}^{*}, \mathrm{a}^{* *}}$ & $62,56^{\mathrm{C}^{*}, \mathrm{a}^{* *}}$ & $55,90^{\mathrm{D}^{*}, \mathrm{a}^{* *}}$ \\
\hline I.S.D & $80,26^{\mathrm{A}^{*}, \mathrm{~b}^{* *}}$ & $60,20^{\mathrm{B}^{*}, \mathrm{~b}^{* *}}$ & $39,72^{C^{*}, b^{* *}}$ & $33,17^{\mathrm{D}^{*}, \mathrm{~b}^{* *}}$ \\
\hline I.D & $63,11^{\mathrm{A}^{*}, \mathrm{c}^{* *}}$ & $47,47^{\mathrm{B}^{*}, \mathrm{c}^{* *}}$ & $31,32^{\mathrm{C}^{*}, \mathrm{c}^{* *}}$ & $24,40^{\mathrm{D}^{*}, \mathrm{c}^{* *}}$ \\
\hline
\end{tabular}

*Médias seguidas por letras maiúsculas iguais nas linhas não diferem entre si pelo teste de Tukey $(p>0,05)$.**Médias seguidas por letras minúsculas iguais nas colunas não diferem entre si pelo teste de Tukey $(p>0,05)$.

Da mesma forma, analisando a viscosidade aparente das amostras em relação à diferença no teor de gordura dos produtos, nota-se que a amostra I.I apresentou maiores valores em comparação às demais em todas as temperaturas de análise, indicando que houve diferença significativa $(p<0,05)$ na $\eta_{A p}$ entre os diferentes tratamentos. Isso demonstra que o teor de gordura está relacionado à $\eta_{\mathrm{Ap}}$ de modo diretamente proporcional, ou seja, para um valor fixo de taxa de deformação, quanto maior o conteúdo de gordura, maior a viscosidade aparente do iogurte, uma vez que essa variação afeta, consequentemente, o teor de sólidos totais do produto que, segundo Antunes et al. (2004), é um fator de grande influência nas características de textura do iogurte.

A relação da viscosidade aparente com a taxa de deformação das amostras pode ser observada pelas curvas de viscosidade na Figura 2. Verifica-se uma redução nos valores de $\eta_{\mathrm{Ap}}$ com o aumento da taxa de deformação dos iogurtes analisados nas quatro temperaturas de trabalho, o que é característico do comportamento de fluidos pseudoplásticos. Sah et al. (2016) encontraram o mesmo comportamento durante a avaliação das propriedades reológicas de iogurte probiótico acrescido de fibras de abacaxi. De acordo com Horne (1998) e Lucey (2002), isso pode ocorrer em razão do enfraquecimento das interações existentes entre as moléculas do produto e da diminuição da energia de interação entre estas.

Verifica-se, nas Figuras, a formação de duas regiões bem distintas para os iogurtes nas quatro temperaturas de análise, que correspondem a comportamentos estruturais bem distintos. Na primeira região, com taxa de deformação inferior a $100 \mathrm{~s}^{-1}$, conforme Bezerra et al. (2013), as forças de agregação, em razão do movimento browniano, começam a ser superadas pelas forças de cisalhamento, induzindo o alinhamento das partículas de modo que a viscosidade aparente dos iogurtes decresce exponencialmente. $\mathrm{Na}$ segunda região, que apresenta valores de taxa de deformação superiores a $100 \mathrm{~s}^{-1}$, o estado de alinhamento e orientação das partículas encontra-se estabelecido e a viscosidade aparente do iogurte tende a ficar constante, sugerindo um comportamento newtoniano. Comportamento semelhante foi observado em outros estudos de caracterização reológica de iogurtes (Mathias et al., 2013; Sah et al., 2016).

Uma análise de regressão não linear foi usada na equação de Arrhenius (Equação 5), a fim de determinar os parâmetros da relação entre $\eta_{\mathrm{Ap}}$ (calculada a uma taxa de deformação de $51,6 \mathrm{~s}^{-1}$ ) e a temperatura, representando de modo satisfatório o efeito da mesma na viscosidade aparente das amostras de iogurte de bacuri $\left(\mathrm{R}^{2}>0,90\right)$, como observado na Figura 3. Constatou-se que, quanto maior a temperatura, menor a viscosidade aparente dos produtos analisados.

Mathias et al. (2013) utilizaram a equação de Arrhenius para avaliar o efeito da temperatura sobre a viscosidade aparente de amostras de iogurtes comerciais com diferentes espessantes. Esses autores verificaram que a curva da amostra IC3 (carboximetilcelulose, goma carragena e goma xantana) não foi bem representada pela equação de Arrhenius, visto que o coeficiente de determinação $\mathrm{R}^{2}$ foi menor que 0,8 , diferente do observado na avaliação dos iogurtes de bacuri.

Os valores da energia de ativação $\left(E_{a t}\right)$ calculados para as amostras analisadas à taxa de deformação fixa de $51,6 \mathrm{~s}^{-1}$ podem ser observados na Tabela 4 . 

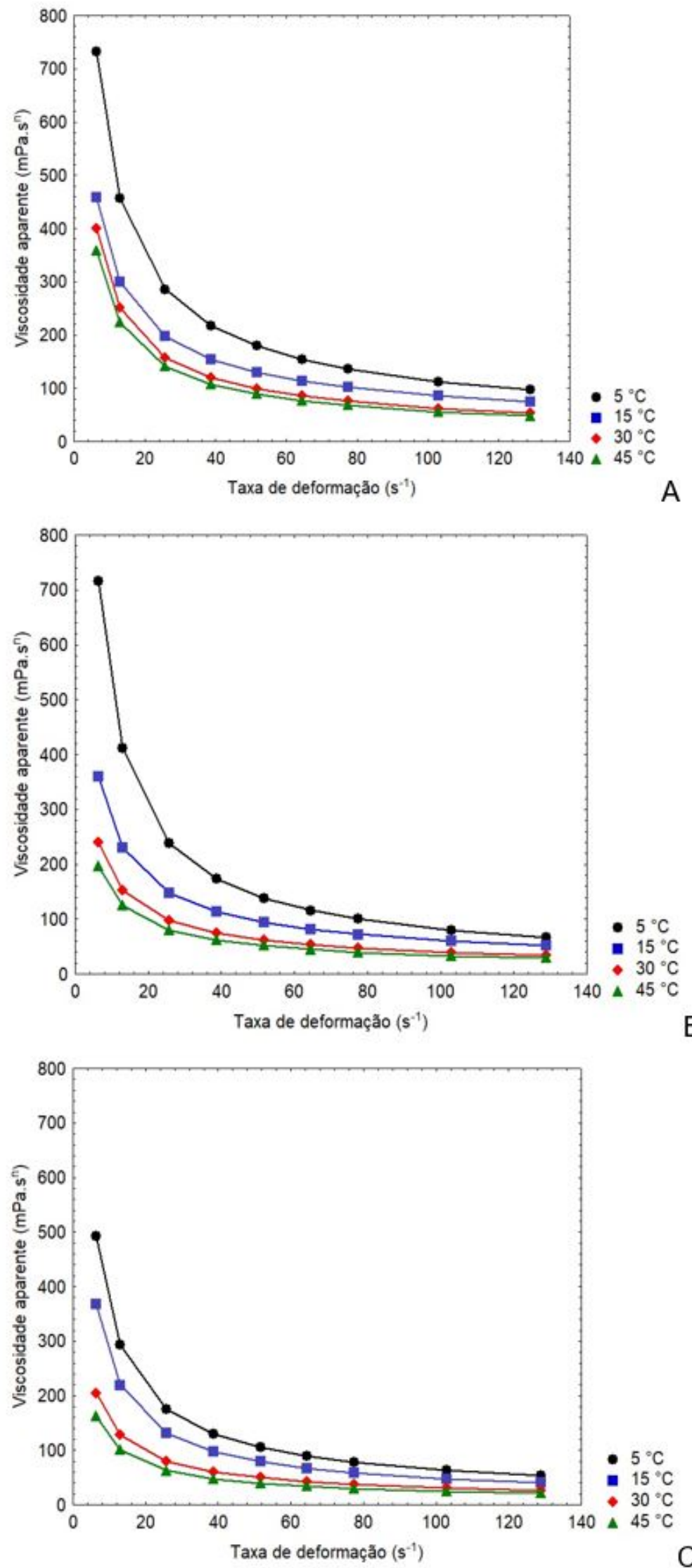

Figura 2. Curvas de viscosidade dos iogurtes de bacuri integral - I.I (A), semidesnatado - I.S.D (B) e desnatado - I.D (C). 


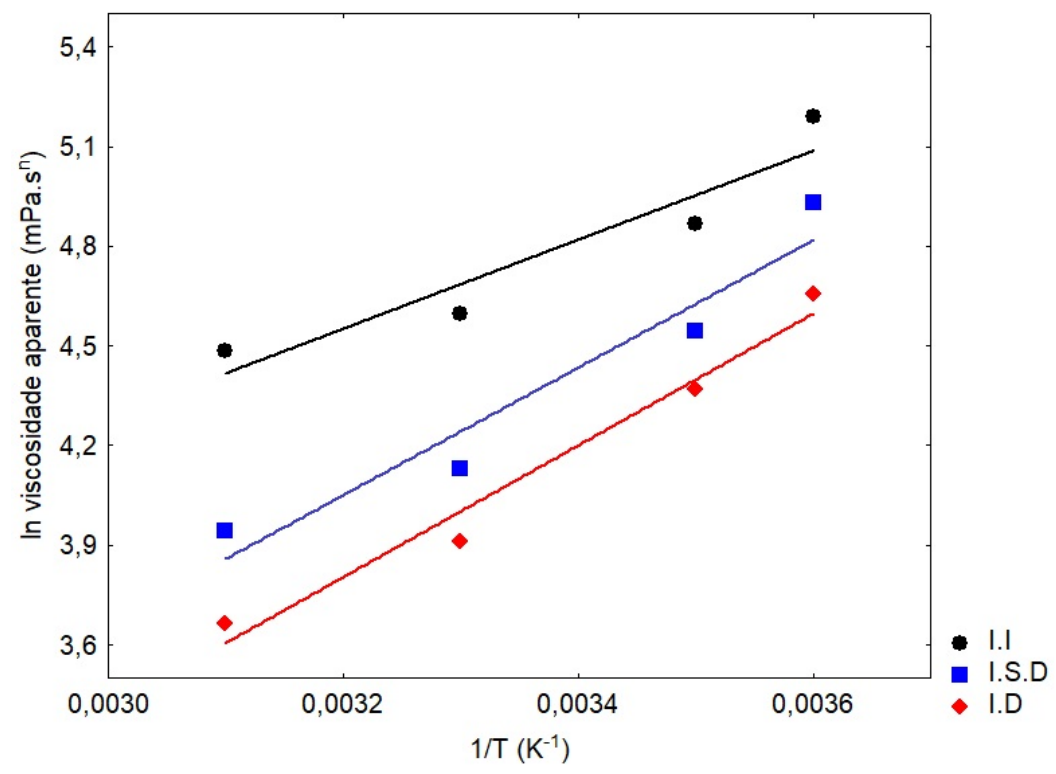

Figura 3. Efeito da temperatura sobre a viscosidade aparente das amostras de iogurte de bacuri integral - I.I, semidesnatado - I.S.D e desnatado - I.D, ajustado pela equação de Arrhenius.

Tabela 4. Energia de ativação das amostras de iogurte de bacuri integral - I.I, semidesnatado - I.S.D e desnatado - I.D.

\begin{tabular}{cccc}
\hline \multirow{2}{*}{$\mathrm{E}_{\mathrm{at}}\left(\mathrm{kcal} \mathrm{g} \mathrm{mol}^{-1}\right)$} & I.I & I.S.D & I.D \\
\cline { 2 - 4 } & $2,66^{\mathrm{b}}$ & $3,81^{\mathrm{a}}$ & $3,93^{\mathrm{a}}$ \\
\hline
\end{tabular}

Médias seguidas por letras iguais não diferem entre si pelo teste de Tukey $(p>0,05)$.

O menor valor de energia de ativação foi obtido para a amostra I.I, enquanto o maior valor correspondeu à amostra I.D, que não mostrou diferença significativa em comparação à amostra I.S.D. Segundo Steffe (1996), em um sistema, elevados valores de $\mathrm{E}_{\mathrm{at}}$ indicam uma mudança mais acelerada da viscosidade aparente com a temperatura. Ao comparar as amostras analisadas, verifica-se que, devido à maior energia de ativação dos materiais semidesnatado e desnatado, essas amostras têm sua estrutura molecular mais influenciada pela temperatura que a outra, ou seja, o iogurte semidesnatado e o desnatado apresentam maior facilidade de escoamento devido à maior energia de ativação.

Esse efeito pode ser justificado pela mudança na estrutura das amostras proveniente da redução no teor de gordura, ocasionando alterações no seu comportamento reológico, fazendo com que sejam mais influenciadas pela mudança de temperatura. Segundo Steffe (1996), os valores da energia de ativação indicam a sensibilidade da viscosidade em função da variação de temperatura: quanto maior o valor de $\mathrm{E}_{\text {at, }}$, mais sensível é o produto a alterações reológicas.

Mathias et al. (2013) encontraram valores de energia de ativação variando entre 2,15 e 6,64 kcal mol-1 e os maiores valores (muito próximos) foram determinados para as amostras IC2 e IC1 (respectivamente 6,64 e 5,50 kcal mol-1), próximos aos obtidos neste trabalho para os iogurtes de bacuri.

\section{Conclusões}

Os resultados demonstraram que os iogurtes elaborados tiveram a viscosidade aparente influenciada pelo teor de gordura e pela variação de temperatura. A redução do teor de gordura do iogurte proporcionou uma mudança nas características relacionadas à textura do material, o que pode proporcionar rejeição por parte do consumidor em comparação ao iogurte integral, tornando interessante a avaliação de um mecanismo capaz de compensar esse efeito. Houve uma redução de $75 \%$ e $79,16 \%$ no teor de gordura dos iogurtes semidesnatado e desnatado, respectivamente, podendo ser considerados produtos com reduzido teor de 
gordura, o que atende às expectativas do consumidor com relação ao fornecimento de alimentos que estimulem a saúde e o bem-estar. O levantamento dos dados reológicos apresentados permite a avaliação preliminar do comportamento dos iogurtes em operações de transporte, agitação e tratamento térmico em nível industrial, possibilitando melhor planejamento de bombas, trocadores de calor e outros equipamentos envolvidos nos processos de produção, aumentando a disponibilidade de dados referentes a produtos desenvolvidos com matérias-primas típicas da Amazônia. Desse modo, a redução do teor de gordura e a adição da polpa de bacuri se mostram convenientes, pois proporcionam a difusão de outros sabores, além dos tradicionalmente oferecidos pelo mercado, e atendem ao consumidor com relação à busca por produtos que exerçam efeitos benéficos ao organismo.

\section{Agradecimentos}

Ao Prof. Dr. Antonio Manoel da Cruz Rodrigues, pela colaboração para a realização deste trabalho.

\section{Referências}

Agyemang, P. N., Akonor, P. T., Tortoe, C., Johnsona, P.-N. T., \& Manu-Aduening, J. (2020). Effect of the use of starches of three new Ghanaian cassava varieties as a thickener on the physicochemical, rheological and sensory properties of yoghurt. Scientific American, 9, 1-10. http://dx.doi.org/10.1016/j.sciaf.2020.e00521

Alpaslan, M., \& Hayta, M. (2002). Rheological and sensory properties of pekmez (Grape molasses)/tahin (Sesame paste) blends. Journal of Food Engineering, 54(1), 89-93. http://dx.doi.org/10.1016/S0260-8774(01)00197-2

Antunes, A. E. C., Cazetto, T. F., \& Bolini, H. M. A. (2004). logurtes desnatados probióticos adicionados de concentrado protéico do soro de leite: perfil de textura, sinérese e análise sensorial. Alimentos e Nutrição, 15(2), 107-114.

Association of Official Analytical Chemists - AOAC. (2016). Official methods of analysis of the Association of the Analytical Chemists (20th ed.) Gaithersburg: AOAC International.

Bezerra, C. V., Silva, L. H. M., Costa, R. D. S., Mattietto, R. A., \& Rodrigues, A. M. C. (2013). Comportamento reológico de suco misto elaborado com frutas tropicais. Brazilian Journal of Food Technology, 16(2), 155-162. http://dx.doi.org/10.1590/S1981-67232013005000020

Bezerra, M. F. (2010). Caracterização físico-química, reológica e sensorial de iogurte obtido pela mistura dos leites bubalino e caprino (Dissertação de mestrado). Universidade Federal do Rio Grande do Norte, Natal. Recuperado em 19 de setembro de 2019, de https://repositorio.ufrn.br/jspui/handle/123456789/15786

Borges, V. C. (2001). Alimentos funcionais: prebióticos, probióticos, fitoquímicos e simbióticos. In D. Waitzberg (Ed.), Nutrição oral, enteral e parenteral na prática clínica (3. ed, pp. 1495-1509). São Paulo: Atheneu.

Brasil. Ministério Agricultura, Pecuária e Abastecimento. (2007, outubro 24). Departamento de Inspeção de Produtos de Origem Animal. Regulamento Técnico de Identidade e Qualidade de Leites Fermentados (Instrução Normativa $n^{\circ} 46$, de 23 de outubro de 2007). Diário Oficial [da] República Federativa do Brasil, Brasília.

Brasil. Agência Nacional de Vigilância Sanitária. (2012, novembro 13). Regulamento Técnico sobre Informação Nutricional Complementar (Resolução da Diretoria Colegiada - RDC n 54, de 12 de novembro de 2012). Diário Oficial [da] República Federativa do Brasil, Brasília.

Chandan, R. C., White, C. H., Kilara, A., \& Hui, Y. H. (2006). Manufacturing yogurt and fermented milks (364 p.). London: Blackwell Publishing Ltda.

Constenla, D. T., Lozano, J. E., \& Crapiste, G. H. (1989). Thermophysical properties of clarified apple juice as a function of concentration and temperature. Journal of Food Science, 54(3), 663-668. http://dx.doi.org/10.1111/j.1365-2621.1989.tb04677.x

Dönmez, O., Mogol, B. A., \& Gökmen, V. (2017). Syneresis and rheological behaviors of set yogurt containing green tea and green coffee powders. Journal of Dairy Science, 100(2), 901-907. PMid:28012628. http://dx.doi.org/10.3168/jds.2016-11262

Fernandes, T. N., Resende, J. V., Cruvinel, S. R. S., \& Reno, M. J. (2010). Relação entre o comportamento reológico e a dinâmica do congelamento e descongelamento de polpa de morango adicionada de sacarose e pectina. Food Science and Technology (Campinas), 30(1), 188-204. http://dx.doi.org/10.1590/S0101-20612010000100029

Fox, P. F., Uniacke-Lowe, T., McSweeney, P. L. H., \& O’Mahony, J. A. (2015). Dairy chemistry and biochemistry (2nd ed., 584 p.). Gewerbestrasse: Springer.

Gonçalvez, D., Pérez, C., Reolon, G., Segura, N., Lema, P., Gámbaro, A., Ares, G., \& Varela, P. (2005). Effect of thickeners on the texture of stirred yogurt. Alimentos e Nutrição, 16(3), 207-211.

Gurjão, F. F., Carneiro, G. G., Pessoa, T., Silva, D. R. S., \& Miranda, D. S. A. (2017). Comportamento reológico de iogurte de cajá comercializado em Campina Grande, Paraíba. Tecnologia \& Ciência Agropecuária, 11(3), 63-67.

Horne, D. S. (1998). Casein interactions: Casting light on the Black Boxes, the structure in dairy products. International Dairy Journal, 8(3), 171-177. http://dx.doi.org/10.1016/S0958-6946(98)00040-5 
Lee, W. J., \& Lucey, J. A. (2010). Formation and physical properties of yogurt. Asian-Australasian Journal of Animal Sciences, 23(9), 1127-1136. http://dx.doi.org/10.5713/ajas.2010.r.05

Lima, S. C. G., Gigante, M. L., \& Almeida, T. C. A. (2006). Efeito da adição de diferentes tipos e concentrações de sólidos nas características sensoriais de iogurte tipo firme. Revista Brasileira de Produtos Agroindustriais, 8(1), 75-84. http://dx.doi.org/10.15871/1517-8595/rbpa.v8n1p75-84

Lucey, J. A. (2002). ADSA Foundation Scholar Award. Formation and physical properties of milk protein gels. Journal of Dairy Science, 85(2), 281-294. PMid:11913691. http://dx.doi.org/10.3168/jds.S0022-0302(02)74078-2

Mathias, T. R. S., Andrade, K. C. S., Rosa, C. L. S., \& Silva, B. A. (2013). Avaliação do comportamento reológico de diferentes iogurtes comerciais. Brazilian Journal of Food Technology, 16(1), 12-20. http://dx.doi.org/10.1590/S1981-67232013005000004

Najgebauer-Lejko, D., Witek, M., Żmudziński, D., \& Ptaszek, A. (2020). Changes in the viscosity, textural properties, and water status in yogurt gel upon supplementation with green and Pu-erh tea. Journal of Dairy Science, 103(12), 11039-11049. PMid:33222847. http://dx.doi.org/10.3168/jds.2020-19032

Özer, B. H., \& Kirmaci, H. A. (2010). Functional milks and dairy beverages. International Journal of Dairy Technology, 63(1), 115. http://dx.doi.org/10.1111/j.1471-0307.2009.00547.x

Penna, A. L. B., Sivieri, K., \& Oliveira, M. N. (2001). Relation between quality and rheological properties of lactic beverages. Journal of Food Engineering, 49(1), 7-13. http://dx.doi.org/10.1016/S0260-8774(00)00179-5

Penna, A. L., Converti, A., \& Oliveira, M. (2006). Simultaneous effects of total solids content, milk base, heat treatment temperature and sample temperature on the rheological properties of plain stirred yogurt. Food Technology and Biotechnology, 44, 515-518.

Ramírez-Sucre, M. O., \& Vélez-Ruiz, J. F. (2013). Physicochemical, rheological and stability characterization of a caramel flavored yogurt. Lebensmittel-Wissenschaft + Technologie, 51(1), 233-241. http://dx.doi.org/10.1016/j.Iwt.2012.09.014

Ribeiro, J. K. G., Soares, F. B., Bezerra, D. S., \& Dimenstein, R. (2007). Níveis de retinol em iogurtes naturais comercializados em supermercados de Natal/RN. Revista do Instituto Adolfo Lutz, 66(2), 120-124.

Ribeiro, M. M., Minim, V. P. R., Minim, L. A., Arruda, A. C., Ceresino, E. B., Carneiro, H. C. F., \& Cipriano, P. A. (2010). Estudo de mercado de iogurte da cidade de Belo Horizonte/MG. Revista Ceres, 57(2), 151-156. http://dx.doi.org/10.1590/S0034$737 X 2010000200003$

Rogez, H., Buxant, R., Mignolet, E., Souza, J. N. S., Silva, E. M., \& Larondelle, Y. (2004). Chemical composition of the pulp of three typical Amazonian fruits: araça-boi (Eugenia stipitata), bacuri (Platonia insignis) and cupuaçu (Theobroma grandiflorum). European Food Research and Technology, 218(4), 380-384. http://dx.doi.org/10.1007/s00217-003-0853-6

Sah, B. N. P., Vasiljevic, T., Mckechnie, S., \& Donkor, O. N. (2016). Physicochemical, textural and rheological properties of probiotic yogurt fortified with fibre-rich pineapple peel powder during refrigerated storage. Lebensmittel-Wissenschaft + Technologie, 65, 978-986. http://dx.doi.org/10.1016/j.Iwt.2015.09.027

Silva, F. C., Guimarães, D. H. P., \& Gasparetto, C. A. (2005). Reologia do suco de acerola: efeitos da concentração e temperatura. Food Science and Technology (Campinas), 25(1), 121-126. http://dx.doi.org/10.1590/S0101-20612005000100020

Silva, V. K. L., Figueiredo, R. W., Brito, E. S., Maia, G. A., Sousa, P. H. M., \& Figueiredo, E. A. T. (2010). Estabilidade da polpa do bacuri (Platonia insignis mart.) congelada por 12 meses. Ciência e Agrotecnologia, 34(5), 1293-1300. http://dx.doi.org/10.1590/S1413-70542010000500030

Soares, D. S., Fai, A. E. C., Oliveira, A. M., Pires, E. M. F., \& Stamford, T. L. M. (2011). Aproveitamento de soro de queijo para produção de iogurte probiótico. Arquivo Brasileiro de Medicina Veterinária e Zootecnia, 63(4), 996-1002. http://dx.doi.org/10.1590/S0102-09352011000400027

Souza, V. A. B., Araujo, E. C. E., Vasconcelos, L. F. L., \& Lima, P. S. C. (2001). Variabilidade das características físicas e químicas de frutos e germoplasma de bacuri da região meio-norte do Brasil. Revista Brasileira de Fruticultura, 23(3), 677-683. http://dx.doi.org/10.1590/S0100-29452001000300047

Steffe, J. F. (1996). Rheological methods in food process engineering (2nd ed., 418 p.). Michigan: Freeman Press.

Teles, C. D., \& Flôres, S. H. (2007a). Influência da adição de espessantes e leite em pó nas características reológicas do iogurte desnatado. Boletim CEPPA, 25(2), 247-256. http://dx.doi.org/10.5380/cep.v25i2.10631

Teles, C. D., \& Flôres, S. H. (2007b). The influence of additives on the rheological and sensory properties of nonfat yogurt. International Journal of Dairy Technology, 60(4), 270-276. http://dx.doi.org/10.1111/j.1471-0307.2007.00354.x

Funding: None. 